\title{
Article \\ Complexity of Molecular Nets: Topological Approach and Descriptive Statistics
}

\author{
Alexander M. Banaru ${ }^{1,2, *}$ and Sergey M. Aksenov $2,3, *$ (D) \\ 1 Faculty of Chemistry, Moscow State University, Vorobievy Hills, 119991 Moscow, Russia \\ 2 Laboratory of Arctic Mineralogy and Materials Sciences, Kola Science Centre, Russian Academy of Sciences, \\ 14 Fersman Str., 184209 Apatity, Russia \\ 3 Geological Institute, Kola Science Centre, Russian Academy of Sciences, 14 Fersman Str., \\ 184209 Apatity, Russia \\ * Correspondence: banaru@mail.ru (A.M.B.); aks.crys@gmail.com (S.M.A.)
}

Citation: Banaru, A.M.; Aksenov, S.M. Complexity of Molecular Nets: Topological Approach and Descriptive Statistics. Symmetry 2022, 14, 220. https://doi.org/10.3390/ sym 14020220

Academic Editor: Enrico Bodo

Received: 5 November 2021

Accepted: 12 January 2022

Published: 24 January 2022

Publisher's Note: MDPI stays neutral with regard to jurisdictional claims in published maps and institutional affiliations.

Copyright: () 2022 by the authors Licensee MDPI, Basel, Switzerland. This article is an open access article distributed under the terms and conditions of the Creative Commons Attribution (CC BY) license (https:// creativecommons.org/licenses/by/ $4.0 /)$.

\begin{abstract}
The molecular net complexity $\left(H_{\text {molNet }}\right)$ is an extension of the combinatorial complexity $\left(H_{\text {mol }}\right)$ of a crystal structure introduced by Krivovichev. It was calculated for a set of 4152 molecular crystal structures with the composition of $\mathrm{C}_{x} \mathrm{H}_{y} \mathrm{O}_{z}$ characterized by the structural class $P 2_{1} / c, \mathrm{Z}=4$ (1). The molecular nets were derived from the molecular Voronoi-Dirichlet Polyhedra (VDP $\mathrm{mol}_{\text {) }}$. The values of the molecular coordination number $\left(\mathrm{CN}_{\mathrm{mol}}\right)$ and critical coordination number $\left(\mathrm{CN}_{\text {crit }}\right)$ are discussed in relation with the complexity of the crystal structures. A statistical distribution of the set of molecular crystals based on the values of $\mathrm{CN}_{\text {mol }}, \mathrm{CN}_{\text {crit }}$, and the complexity parameters is obtained. More than a half of the considered structures has $\mathrm{CN}_{\text {mol }}=14$ and $\mathrm{CN}_{\text {mol }}{ }^{\prime}=9$ with the Wyckoff set of edges $e^{5} d c b a$. The average multiplicity of intermolecular contacts statistically significantly decreases from 1.58 to 1.51 upon excluding all contacts except those bearing the molecular net. The normalized value of $H_{\text {molNet }}$ is of the logistic distribution type and is distributed near $0.85 \mathrm{H}_{\text {molNet }}$ with a small standard deviation. The contribution of $H_{\text {mol }}$ into $H_{\text {molNet }}$ ranges from 35 to $95 \%$ (mean $79 \%$, SD $6 \%$ ), and the subset of bearing intermolecular contacts accounts for 41 to $100 \%$ (mean $62 \%$, SD 11\%) of the complexity of the full set of intermolecular contacts.
\end{abstract}

Keywords: information measure; complexity; crystal structure; crystallographic net; coordination number

\section{Introduction}

According to the approach initially developed by Shannon in his theory of communication [1], the complexity of a message consisting of symbols depends on the probability of occurrence of each symbol in the message. In particular, quantifying information content of a message in bits corresponds as the function:

$$
\begin{gathered}
H=\sum_{i=1}^{k} L\left(p_{i}\right) \\
L\left(p_{i}\right)=\left\{\begin{array}{c}
0\left(p_{i}=0\right), \\
-p_{i} \log _{2} p_{i}\left(p_{i}>0\right)
\end{array}\right.
\end{gathered}
$$

where $p_{i}$ is the probability of $i$-th symbol to appear in the given message. Any graph with certain types of vertices may be considered as a message, as well. Finite graphs corresponding to the molecules belong to a wide class of so-called chemical graphs, and the approaches of measuring information content for them were introduced in the 1950s by Trucco [2] and in the early 2000s reviewed by Bonchev [3]. Commonly, the vertices of a chemical graph $G$ are referred to as equivalent if they belong to the same orbit of the automorphism group of the graph $\operatorname{Aut}(G)$, which is isomorphic to the maximal symmetry group of the graph. The information measures of molecules and their ensembles was 
recently reviewed by Sabirov and Shepelevich [4]. Information content of molecules is of a specific interest due to the studying of chemical reactions [5], molecular aggregation processes [6], searching the reason for the first bioorganic molecules to appear [7], etc.

A crystal structure can also be represented by a finite graph called quotient graph introduced by Chung et al. in the 1980s [8]. In fact, the quotient graph is a "molecule" of a non-molecular crystal. A quotient graph of the crystal structure maps atoms onto the vertices and chemical bonds onto the edges or loops and reflects the connectivity of the reduced unit cell of the structure. The quotient graph is a useful tool to enumerate nets occurring in crystal structures [9] and perform a topological analysis of underlying nets $[10,11]$. The cyclomatic number of the quotient graph equals the dimensionality $n$ of the Euclidean space $E^{n}$ in which the net derived from the quotient graph may be embedded being periodic in the same number of linearly independent directions. In such a space, the deletion of any edge lattice of the net leads to a disconnected net, and the net is referred to as minimal [12-14]. For instance, the diamondoid net is minimal in $E^{3}$, while the quartz net is minimal in $E^{4}$. Embeddings of some typical nets in $E^{3}$ were enumerated in Reticular Chemistry Structure Resource (RCSR) [15], where each net is characterized by the maximal possible symmetry achieved by a barycentric placement of the vertices $[16,17]$.

The amount of information stored by the quotient graph of the crystal structure was introduced by Krivovichev $[18,19]$ to quantify the information content of the crystal. In this case, the probability $p_{i}$ from (2) is calculated as $p_{i}=m_{i} / v$, where $m_{i}$ is the multiplicity of the $i$-th crystallographic orbit occupied by atoms; $v$ - the number of atoms in the reduced unit cell. Later, Hornfeck [20] complemented this measure by terms considering the degrees of freedom associated with a translational motion of an atom along the Wyckoff position and Kaußler and Kieslich [21] adapted this measure to positionally disordered crystals. However, for molecular crystals the information content calculated using this approach indicates the complexity of the molecule itself instead of the crystal structure. The possible scheme of avoiding this problem was proposed in [22]:

$$
\begin{gathered}
H_{\text {molNet }}=H\left(2 N, \mathrm{CN}_{\mathrm{mol}}\right)+\frac{2 N}{2 N+\mathrm{CN}_{\mathrm{mol}}} H_{\mathrm{mol}}+\frac{\mathrm{CN}_{\text {mol }}}{2 N+\mathrm{CN}_{\mathrm{mol}}} H_{\text {edge }} ; \\
H\left(2 \mathrm{~N}, \mathrm{CN}_{\mathrm{mol}}\right)=-\frac{2 N}{2 N+\mathrm{CN}_{\mathrm{mol}}} \log _{2} \frac{2 N}{2 N+\mathrm{CN}_{\mathrm{mol}}}-\frac{\mathrm{CN}_{\mathrm{mol}}}{2 N+\mathrm{CN}_{\mathrm{mol}}} \log _{2} \frac{\mathrm{CN}_{\mathrm{mol}}}{2 N+\mathrm{CN}_{\mathrm{mol}}} ; \\
H_{\text {molNet, tot }}=\left(N+\mathrm{CN}_{\mathrm{mol}} / 2\right) H_{\text {molNet }}
\end{gathered}
$$

where $N$ is the number of atoms in the molecule, $\mathrm{CN}_{\text {mol }}$-the molecular coordination number, $H_{\text {edge }}$ - the information content of the molecule, $H_{\text {edge }}$ - the information content of the edge net of the molecular net, $H_{\text {molNet }}$ - the combination of $H_{\text {mol }}$ and $H_{\text {edge }}$ with the property of strong additivity $[20,23]$. The value of $H_{\text {molNet }}$ is meaningful even for high symmetric molecular structures with the only orbit occupied by the atoms (i.e., $\mathrm{I}_{2}, \mathrm{~S}_{6}$, and $\alpha-\mathrm{N}_{2}$ ) [22]. It should be noted that the molecule in the crystal structure is commonly distorted, and the only symmetry operation retained in a molecule (in more than $90 \%$ cases) is the inversion center [24], which requires for the preserving of dense packing according to Kitaigorodskii [25]. Generally, a more conformational lability of the molecule promotes a more diverse set of contacts in the coordination shell and should result in the increasing of the molecular net complexity. On the other hand, certain molecular fragments have the opportunity to form a specific intermolecular interaction, such as $\mathrm{H}$-bonds, $\pi \ldots \pi$ interactions, Hal ... Hal, etc. In such a case the small subset of interactions often predominates in the crystal structure and, in fact, is bearing the entire net of the intermolecular contacts. However, the subset of bearing contacts may include excessive interactions and thus be redundant. The portion of the bearing subnet complexity attributable to the target engineered interactions may serve an indicator of effectiveness of a crystal engineering technique, as the latter aims to reproduce targeted bearing contacts.

In this work the formalism (3)-(5) previously discussed in [26] is tested for the set of more than 4000 homomolecular crystals with the general formula $\mathrm{C}_{x} \mathrm{H}_{y} \mathrm{O}_{z}$ of a structural 
class $P 2_{1} / c, Z=4(1)$ (such notation indicates that there is exactly one symmetrically unique molecule occupying a general orbit in the space group $P 2_{1} / c$ ). This structural class is of the special interest as the most widespread among organic crystals and corresponding to $\sim 1 / 3$ of all homomolecular structures and more than $1 / 2$ of homomolecular racemates [27]. The aim of this work is to investigate the partitioning of intermolecular contacts from the coordination shell of the molecule into equivalence classes and to obtain the distribution type and the descriptive statistics of $H_{\text {molNet }}$.

\section{Methods}

The initial set of the crystal structures was extracted from Cambridge Structural Database (CSD) [28] using the following restrains: the presence of atomic coordinates, the absence of errors and/or disorder, and $R$-factor $<5 \%$. Out of 4249 high-quality molecular crystal structures [26] selected from CSD ver. 5.41 (with updates), the set of 4152 structures without duplicates was retained for further investigation. The criteria of considering a structure as a duplicate were the same cell dimensions (with the tolerance of $2 \sigma$ ), the same chemical composition, space group and Wyckoff sequence.

The construction of molecular nets was carried out using the ToposPro program [29] by calculating the solid angles of the molecular Voronoi-Dirichlet polyhedron $\left(\mathrm{VDP}_{\mathrm{mol}}\right)$. According to Blatov [30], $\mathrm{VDP}_{\mathrm{mol}}$ is the superposition of atomic VDPs in a molecule, and the solid angle $(\Omega)$ corresponding to an intermolecular contact arises from interatomic contacts as:

$$
\Omega=\frac{\sum \Omega_{i j}}{\Omega_{\Sigma}} \times 100 \%
$$

where $\Omega_{i j}$ is the solid angle for the intermolecular contact $i j$, and $\Omega_{\Sigma}$-the sum of solid angles for all the interatomic contacts for the given molecule with the adjacent ones. Interatomic contacts with $\Omega_{i j}<1.5 \%$ of $4 \pi$ steradians are omitted. In the same way, intermolecular contacts with $\Omega<1.5 \%$ in this work have been omitted, while the adjacent molecules with $\Omega \geq 1.5 \%$ are considered as the coordination shell of the initial molecule (Figure 1). As a rule, for non-specific van der Waals interactions the descending order of $\Omega$ corresponds to the decrease of interaction energy, allowing to avoid energy calculations for the assessment of supramolecular arrangement [26].

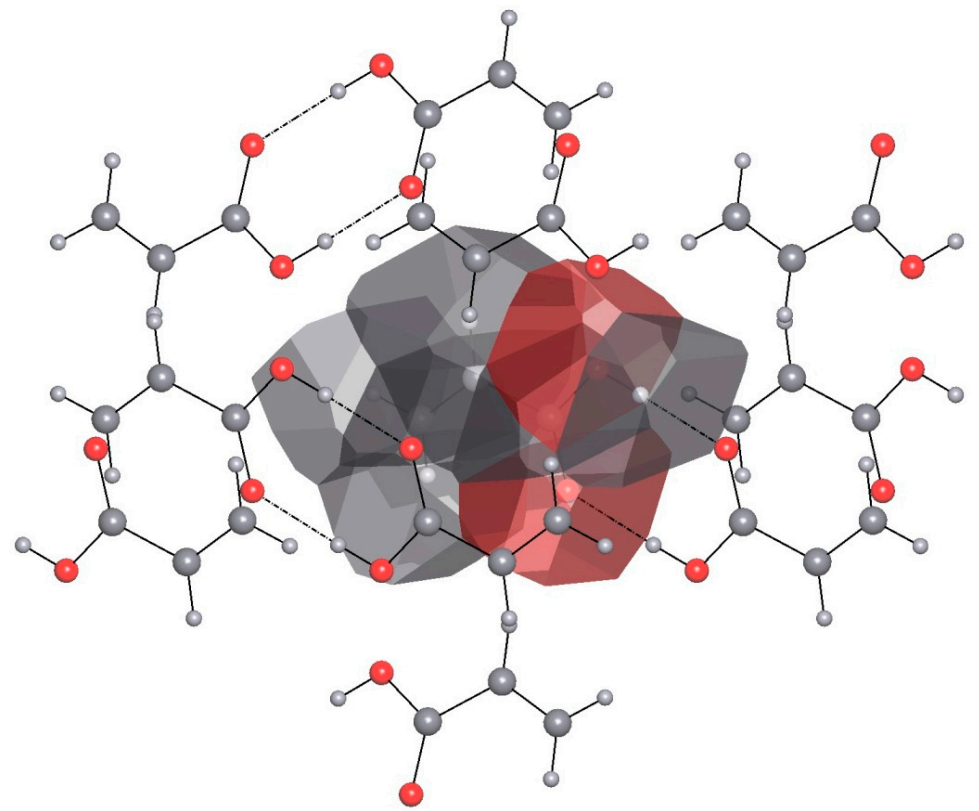

Figure 1. VDP $\mathrm{mol}_{\text {as }}$ as the sum of atomic VDPs (represented by different colors) and the 1-st molecular coordination shell in the crystal structure of acrylic acid (CSD-refcode: ACRLAC04). 
To derive the molecular net, the atoms were pulled to the mass center of the molecule. The molecular coordination number $\left(\mathrm{CN}_{\text {mol }}\right)$, which includes only symmetrically independent intermolecular contacts, is marked by a prime $\left(\mathrm{CN}_{\text {mol }}\right.$ '), i.e., acrylic acid (ACRLAC04 [31]) has $\mathrm{CN}_{\text {mol }}=12$ (cuboctahedron) and $\mathrm{CN}_{\text {mol }}{ }^{\prime}=8$. The subset of bearing contacts generating so-called critical net for a given molecule was defined in [32]. In a monosystemic crystal structure the center of gravity of each molecule is connected with the centers of gravity of $\mathrm{CN}_{\text {mol }}$ adjacent molecules, and VDP faces have the following order of the solid angles: $\Omega_{1}>\Omega_{2}>\Omega_{3}>\ldots>\Omega_{n}$ (symmetrically equivalent contacts have the same $\Omega$ ). For any value of $n$, there is $1 \leq k \leq n$ such that if all edges corresponding to the solid angles $\Omega_{k}, \Omega_{k+1}, \ldots, \Omega_{n}$ are removed from the net, the resulted net becomes disconnected. The value $\max (k)$ is called a "critical coordination number with a prime" $\left(\mathrm{CN}_{\text {crit }}{ }^{\prime}\right)$. If all symmetrically equivalent contacts are considered, the corresponding value is called a "critical coordination number without a prime" $\left(\mathrm{CN}_{\mathrm{mol}}\right)$. For instance, acrylic acid (ACRLAC04) has $\mathrm{CN}_{\text {crit }}=5$ (square pyramid) and $\mathrm{CN}_{\text {mol }}{ }^{\prime}=4$. To derive a $\mathrm{CN}_{\text {crit }}$, firstly, the edges of the net of intermolecular contacts, for which $\Omega>15 \%$, were removed from the net. In all cases, this led to reduction of the net's dimensionality from 3D to 2D, 1D, or 0D. Then the contacts with $\Omega=14.5-15.0 \%$ were returned to the adjacency matrix of the centers of gravity of the molecules, and a check was performed to establish the dimensionality of the net again. If the dimensionality was 3D, the returned contacts were referred as $\Omega_{\text {crit }}=\Omega_{\max (k)}$, and the constructed 3D net was considered a net of bearing contacts. If the dimensionality of the net did not increase to 3D, then the contacts with $\Omega=14.0-14.5 \%$ were added to the adjacency matrix, and the dimensionality of the net was checked again. This procedure was repeated with the step of $0.5 \%$ until $\Omega_{\text {crit }}$ was found for all the structures. The less step values are not reliable since the measurement error is about $0.5 \%$. The obtained distribution of the crystal structures of the considered set is close to normal (Figure 2).

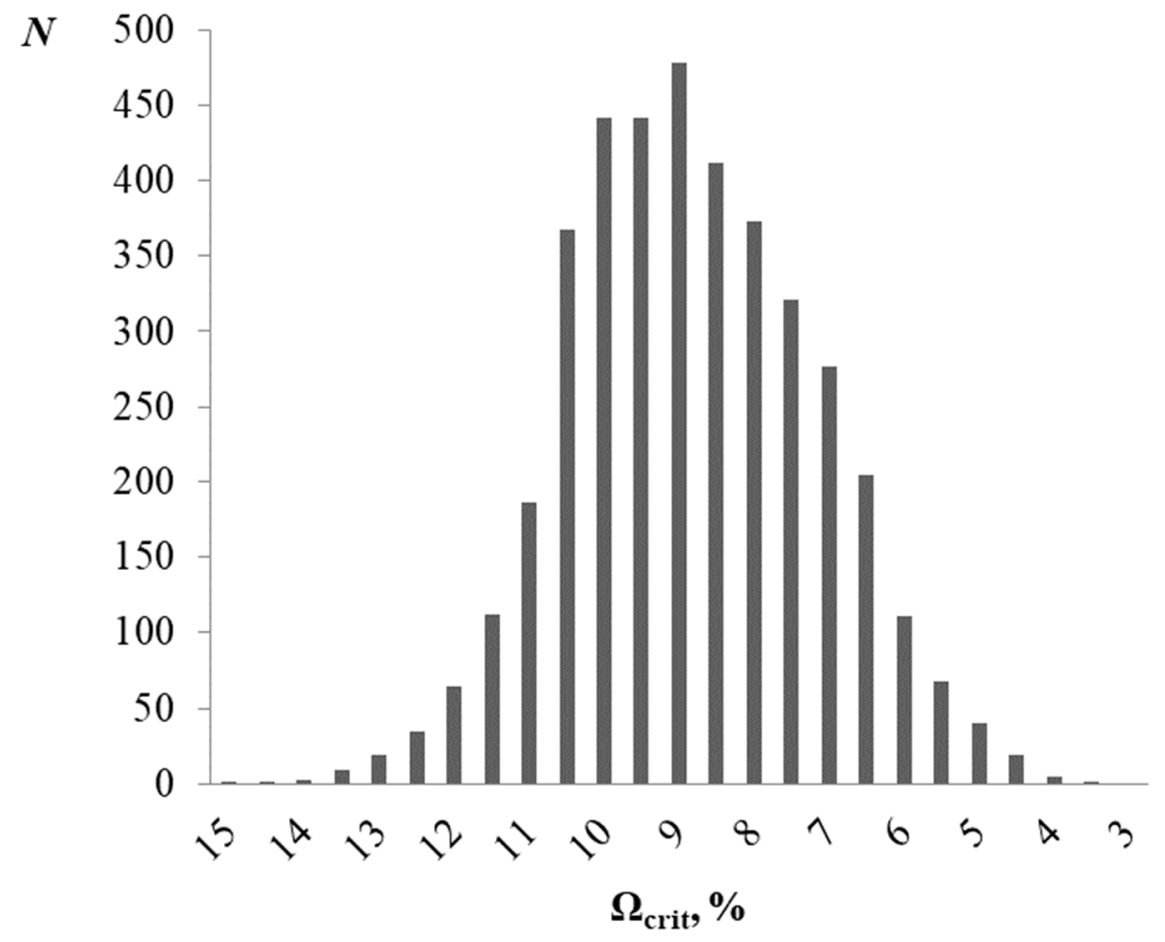

Figure 2. The distribution of the set of crystal structures by $\Omega_{\text {crit }}$ rounded to half-integer $\%$.

The nets of intermolecular contacts in the most symmetrical embedding in $E^{3}$ are classified either in accordance with RCSR [15] or TopCryst database [33] (when RCSR classification is lacking). The nets those remain unclassified in RCSR and TopCryst database up to date are characterized by a point symbol. The net for the crystal structure of acrylic acid has the RCSR code fcu (cubic closest packing), while the net of bearing contacts-sqp 
(Figure 3). For a $\mathrm{CN}$-coordinated net there are $\mathrm{CN}(\mathrm{CN}-1) / 2$ angles. The shortest cycle in each angle should be identified. The point symbol in the form $A^{a} \cdot B^{b} \ldots C^{c}$ indicates that there are $a$ angles that are $A$-cycles, $b$ angles that are $B$-cycles, etc. $(A<B<\ldots<C)$ [34]. For instance, the fcu net has $12 \cdot 11 / 2=66$ angles in each vertex, and its point symbol is $3^{24} \cdot 4^{36} \cdot 5^{6}$, while the sqp net has $5 \cdot 4 / 2=10$ angles in each vertex, and its point symbol is $4^{4} \cdot 6^{6}$.
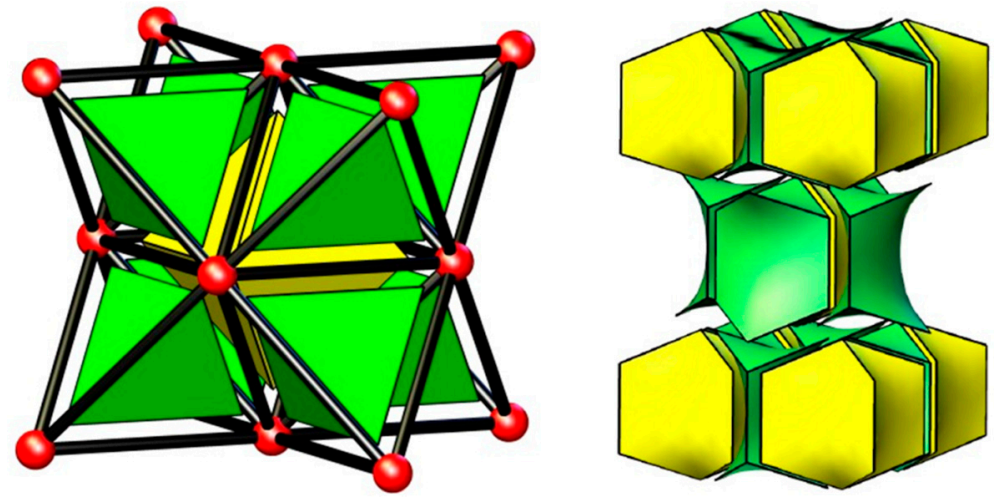

Figure 3. A fragment of the net of all intermolecular contacts fcu type (left) and that of bearing intermolecular contacts sqp type (right) for the crystal structure of acrylic acid.

If there are $p$ sorts of vertices and $q$ sorts of edges in the net, then the net is called $p, q$-transitive. For instance, the fcu and sqp nets are 1,1 and 1,2-transitive, respectively. In fact, $p$ and $q$ denote the minimal number of orbits occupied by the molecular centers of gravity and the contacts, respectively, and interrelate with the molecular net complexity for its most symmetric embedding in $E^{3}$.

The complexity of a molecular net was calculated using (3)-(5). The structural information content (SIC $=0-1$ ) [4] meaning the same as a normalized informational complexity [19] and was calculated as follows:

$$
\mathrm{SIC}=H / \max (H)
$$

where $\max (H)$ is the maximal possible value of $H$, when each vertex constitutes its own equivalence class: $\max \left(H_{\mathrm{mol}}\right)=\log _{2} N ; \max \left(H_{\text {edge }}\right)=\log _{2} \mathrm{CN}_{\text {mol }} ; \max \left(H_{\text {molNet }}\right)=\log _{2}\left(2 N+\mathrm{CN}_{\text {mol }}\right)$.

The molecule of acrylic acid has $N=9$ atoms and all of them are symmetrically unique (the Wyckoff set $e^{9}$ in the space group $\left.P 2_{1} / c\right), m_{i}=4, v=36$. Consequently, $H_{\text {mol }}=-9 \cdot 4 / 36 \cdot \log _{2}(4 / 36)=3.170$ bits/atom. The edge net of the $\mathrm{CN}_{\text {mol-coordinated }}$ molecular net is generated by the midpoints added to each edge of the molecular net. Two midpoints are connected if and only if they are adjacent to the same vertex, and the vertices of the initial net are removed. The final net (edge net) is $2\left(\mathrm{CN}_{\mathrm{mol}}-1\right)=\mathrm{CN}_{\text {edge }}$-connected . The edge net for acrylic acid is 22-connected and contains 8 symmetrically independent vertices with the Wyckoff sequence $e^{4} d c b a, v=24, H_{\text {edge }}=-16 / 24 \cdot \log _{2}(16 / 24)-$ $4 \cdot 2 / 24 \cdot \log _{2}(2 / 24)=2.918$ bits $/$ contact; $H\left(2 N, \mathrm{CN}_{\mathrm{mol}}\right)=H(18,12)=0.971$ bits $/$ d.f. (per a degree of freedom), $H_{\text {molNet }}=4.040$ bits $/$ d.f., $\mathrm{SIC}_{\text {molNet }}=0.823, H_{\text {molNet,tot }}=4.040 \cdot 15=$ 60.60 bits/molecule. Note that if just bearing contacts are included in the net, then the edge net would be 8 -connected and contain only 4 of 8 independent vertices with the Wyckoff sequence $e d c a, v=10, H_{\text {edge }}=-4 / 10 \cdot \log _{2}(4 / 10)-3 \cdot 2 / 10 \cdot \log _{2}(2 / 10)=1.922$ bits $/$ contact. This net is characterized by the unknown topological type.

The discriminatory power of $H$, based on the probability of two unrelated objects being characterized as the same type, was calculated according to the following equation [35]:

$$
D=1-\frac{1}{N(N-1)} \sum_{j=1}^{s} x_{j}\left(x_{j}-1\right)
$$

where $N$ is the number of the tested crystal structures, $s$ the number of different types of structures with respect to $H$, and $x_{j}$ the number of objects belonging to the $j$-th type. 
The correlations between calculated values were sought in the Mathematica software ver. 11.0 [36].

\section{Results and Discussion}

Crystal structures of the analyzed set are distributed over $\mathrm{CN}_{\mathrm{mol}}$, generally, in accordance with the earlier results obtained by Carugo et al. [37]. More than a half of the crystal structures have $\mathrm{CN}_{\text {mol }}=14$, and the second ranked value $\mathrm{CN}_{\text {mol }}=16$. The most frequent values of $\mathrm{CN}_{\text {crit }}$ are 5, 4, and 6, but unlike $\mathrm{CN}_{\text {mol }}$ there is no sharp peak on any of the values (Figure 4). More than a half of the structures is characterized by $\mathrm{CN}_{\text {mol }}{ }^{\prime}=9$ (with $e^{5}-2355$ structures; with $e^{6}-84$ structures; with $e^{4}-13$ structures; $e^{7} b a-1$ structure with refcode HINSOM [38]), and most abundant $\mathrm{CN}_{\text {crit }}{ }^{\prime}$ is its least value 3 (eba, ecb, $e-856$ structures; $e^{2} a, e^{2} b, e^{2}-737$ structures; $e^{3}-81$ structures).
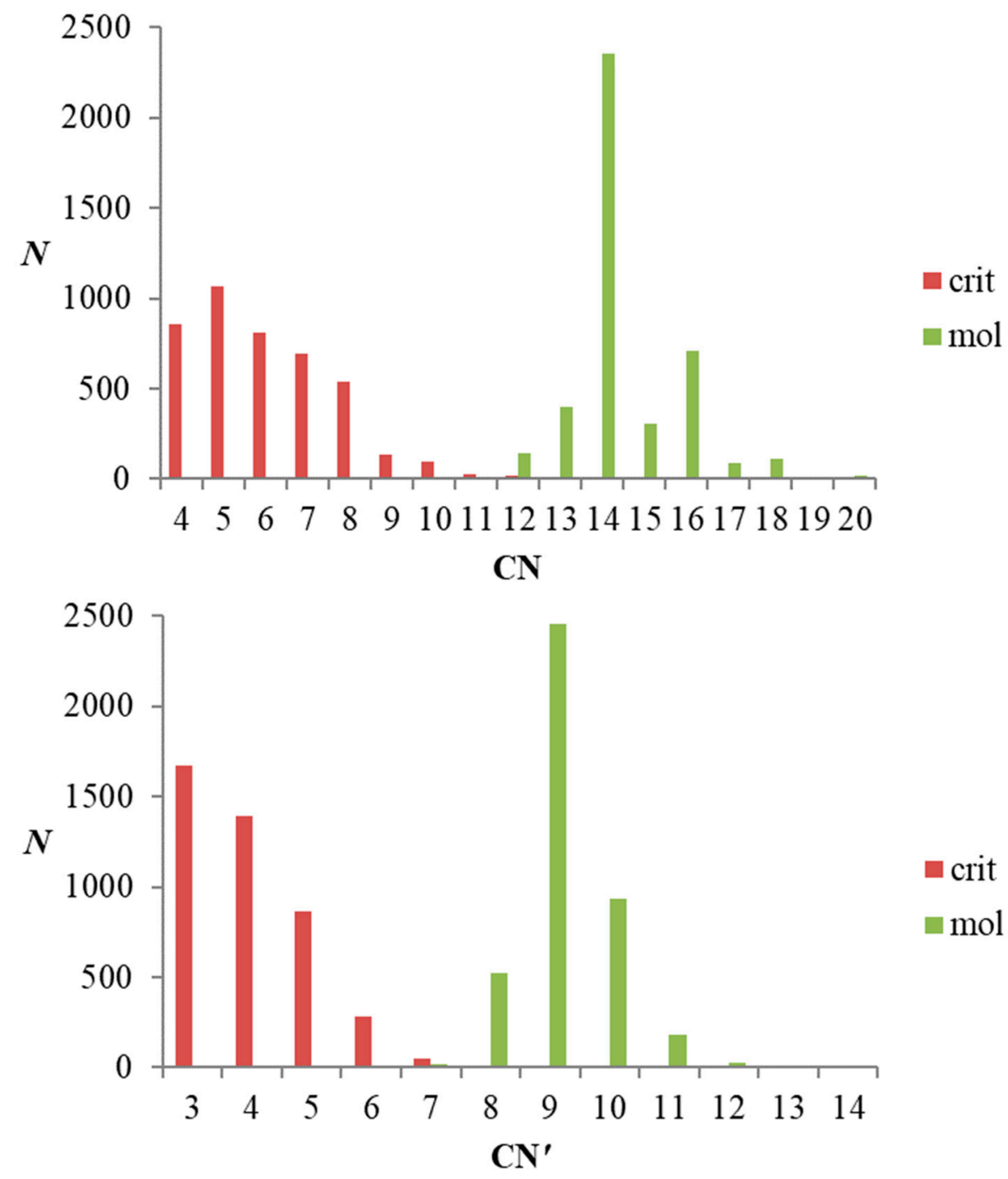

Figure 4. The distribution of crystal structures of the analyzed set over $\mathrm{CN}_{\mathrm{mol}}$ and $\mathrm{CN}_{\text {crit }}$ (top), $\mathrm{CN}_{\text {mol }}$ ' and $\mathrm{CN}_{\text {crit }}{ }^{\prime}$ (bottom).

In the structural class $P 2_{1} / c, Z=4(1)$ each molecule can form contacts with a multiplicity 1 or 2 . The former corresponds to a so-called involution, a symmetry element of the order 2 (the midpoint of a contact occupies the Wyckoff position $e$ ). The only involution presence in the space group $P 2_{1} / c$ is the inversion center $\overline{1}$ (the midpoint of a contact occupies the Wyckoff position $a, b, c$ or $d$ ). All other contacts are formed via a screw axis $2{ }_{1}$, or a glide plane $c$, or a translation along some direction. It is easy to show that the average multiplicity in between 1 and 2 equals to $v / 2 \mathrm{CN}^{\prime}$. According to two-sample $t$-test, the 
difference of the mean values for all contacts and for those bearing the net is statistically significant ( $p$-value $<0.001$ ). Moreover, the minimal multiplicity is 1.375 ( 3 structures) for the hole net of molecular contacts unlike 1.000 (1 structure with refcode KOLRAF [39]) for the critical subnet (Table 1). That is why the subnet of bearing contacts is, in average, more enriched by the inversion centers than the hole molecular net. As shown above, 2355 structures have the Wyckoff sequence $e^{5} d c b a$ (or similar) for the edge net, and mean multiplicity in this case is $(5 \cdot 2+4) / 9=1.556$. Motherwell [40] previously studied the projection patterns formed by projecting coordination shell of a molecule into 2D in different space groups with none of the special positions occupied. The majority of projection patterns in $P 2_{1} / \mathrm{c}$ contained at least one contact via an inversion center.

Table 1. The min, max, mean value, and standard deviation of the multiplicity of the contact in the molecular net of all the intermolecular contacts (molecular) and the bearing ones only (critical) for the set of 4152 crystal structures.

\begin{tabular}{ccccc}
\hline The Net & Min & Max & Mean & б \\
\hline molecular & 1.375 & 2.000 & 1.582 & 0.060 \\
critical & 1.000 & 2.000 & 1.512 & 0.186 \\
\hline
\end{tabular}

The distribution of molecular nets in the considered series over the topological types is, generally, in accordance with the trend previously found by Carugo et al. for 105549 packings of small molecules [37]. The most widespread topological type is bcu-x, a type derived from the body-centered cubic lattice where the coordination shell of the atom is extended by the second coordination shell $\left(\mathrm{CN}_{\mathrm{mol}}=8+6\right)$. This topological type has the least topological density $\mathrm{TD}_{10}$ that reflects the total number of vertices in the first 10 of coordination shells, among all 14-coordinated nets reported for centrosymmetric [41] and non-centrosymmetric [32] crystalline hydrocarbons, some inorganic molecular crystals [22] (i.e., 14T191 in the orthorhombic sulfur, $\alpha-S_{8}$ ), and those with the most popularity amongst all small molecular crystals [37]. Recently studied crystal structure of 2-(tert-butyl)-4-chloro6-phenyl-1,3,5-triazine with 2 symmetrically independent molecules [42] is characterized by the 14T319 type topology (after neglecting contacts with $\Omega \leq 2 \%$ ), which occupies the opposite side of 14-coordinated molecular nets with respect to $\mathrm{TD}_{10}$ (Table 2). The more 2nd or 3rd CN does not mean the more 4th and 5th CNs. For instance, in the 2nd coordination sphere there are 54 vertices in 14T134 topological type and 53 in 14T10; nevertheless, $\mathrm{TD}_{10}$ for 14T10 is slightly higher. Remark that the TopCryst database has been extended last years by many new topological types with large $\mathrm{CNs}$, including $\mathrm{CN}=14$. Thus, the previously found in 2019 a 14T134 topological type [32] in the crystal structure of spiropentane (refcode VAJGOC [43]) has no reference code in the TopCryst database. The corresponding molecular net in the most symmetric embedding in $E^{3}$ is 1,6-transitive and has the space group $R \overline{3} c$ with the only general position occupied by centers of gravity of molecules.

Consider three typical examples of molecular nets realized in $\alpha$-methyl-trans-cinnamic acid (refcode: BEJVOB [45]), 5-methoxyindan-1-one (refcode: KACSOX01 [46]), which are both isomers with the chemical formula $\mathrm{C}_{10} \mathrm{H}_{10} \mathrm{O}_{2}$ (Figure 5), and (1RS,3SR,4SR)trispiro(2.0.0.2.1.1)nonane-1-carboxylic acid with the chemical formula $\mathrm{C}_{10} \mathrm{H}_{12} \mathrm{O}_{2}$ (refcode: FAFDEW [47]). The Wyckoff sequences for the molecules are: $e^{22}$ for BEJVOB and KACSOX01, and $e^{24}$ for FAFDEW. This leads to a slightly different values of $H_{\mathrm{mol}}: H_{\mathrm{mol}}=4.459$ bits $/$ atom for BEJVOB and KACSOX01, and $H_{\mathrm{mol}}=4.585$ bits $/$ atom for FAFDEW. All other structures from the set of 4152 structural files show exactly the same distribution of atoms over general positions, i.e., they have the maximal $H_{\mathrm{mol}}$ for the given $N(\mathrm{SIC}=1)$. Indeed, if a molecular center of gravity occupies a general position, then no atom is able to occupy an inversion center, otherwise the other atoms should be related by the inversion center and the molecule would either occupy the special position or have a symmetry-induced disorder (the latter was restricted by the structure selection). The linear correlation coefficient between $N$ and the molecular mass in the analyzed set is 0.936 , between $N$ and $H_{\mathrm{mol}}-0.959$, and between the molecular mass and $H_{\mathrm{mol}}-0.889$. 
Consequently, there is a strong positive linear correlation between the molecular mass, $N$, and $H_{\mathrm{mol}}$.

Table 2. The topological types found in crystalline hydrocarbons [32,44], some inorganic molecular crystals [22], and 2-(tert-butyl)-4-chloro-6-phenyl-1,3,5-triazine, ordered by the increase of $\mathrm{TD}_{10}$.

\begin{tabular}{cccccccc}
\hline \multirow{2}{*}{ Topological Type } & \multirow{7}{*}{ Point Symbol } & \multicolumn{5}{c}{ Coordination Sphere } & \multirow{2}{*}{ TD $_{\mathbf{1 0}}$} \\
\cline { 3 - 6 } & & 1st & 2nd & 3rd & 4th & 5th & \\
\hline bcu-x & $3^{36} \cdot 4^{48} \cdot 5^{7}$ & 14 & 50 & 110 & 194 & 302 & 4641 \\
gpu-x & $3^{36} \cdot 4^{46} \cdot 5^{9}$ & 14 & 52 & 114 & 202 & 314 & 4831 \\
tcg-x & $3^{36} \cdot 4^{46} \cdot 5^{9}$ & 14 & 52 & 116 & 204 & 318 & 4893 \\
$\mathbf{1 4 T 3 4}$ & $3^{33} \cdot 4^{51} \cdot 5^{7}$ & 14 & 53 & 117 & 208 & 324 & 4996 \\
$\mathbf{1 4 T 5}$ & $3^{36} \cdot 4^{45} \cdot 5^{10}$ & 14 & 53 & 120 & 212 & 332 & 5106 \\
$\mathbf{1 4 T 6}$ & $3^{36} \cdot 4^{45} \cdot 5^{10}$ & 14 & 53 & 120 & 213 & 335 & 5138 \\
$\mathbf{1 4 T 1 3 4}$ & $3^{34} \cdot 4^{47} \cdot 5^{10}$ & 14 & 54 & 122 & 216 & 338 & 5201 \\
$\mathbf{1 4 T 1 0}$ & $3^{36} \cdot 4^{45} \cdot 5^{10}$ & 14 & 53 & 122 & 218 & 339 & 5238 \\
$\mathbf{1 4 T 3 7}$ & $3^{33} \cdot 4^{45} \cdot 5^{10}$ & 14 & 53 & 121 & 217 & 339 & 5239 \\
$\mathbf{1 4 T 6 5}$ & $3^{33} \cdot 4^{51} \cdot 5^{7}$ & 14 & 54 & 122 & 218 & 342 & 5301 \\
$\mathbf{1 4 T 9}$ & $3^{36} \cdot 4^{45} \cdot 5^{10}$ & 14 & 53 & 123 & 221 & 344 & 5329 \\
$\mathbf{1 4 T 2 4}$ & $3^{36} \cdot 4^{46} \cdot 5^{9}$ & 14 & 52 & 120 & 218 & 344 & 5339 \\
$\mathbf{1 4 T 3}$ & $3^{36} \cdot 4^{44} \cdot 5^{11}$ & 14 & 54 & 124 & 222 & 348 & 5373 \\
$\mathbf{1 4 T 8}$ & $3^{36} \cdot 4^{44} \cdot 5^{11}$ & 14 & 54 & 126 & 226 & 354 & 5475 \\
$\mathbf{1 4 T 3 1 9}$ & $3^{30} \cdot 4^{50} \cdot 5^{11}$ & 14 & 58 & 130 & 232 & 362 & 5581 \\
$\mathbf{1 4 T 1 8}$ & $3^{36} \cdot 4^{44} \cdot 5^{11}$ & 14 & 54 & 130 & 242 & 382 & 5947 \\
$\mathbf{1 4 T 1 9 1}$ & $3^{33} \cdot 4^{47} \cdot 5^{11}$ & 14 & 59 & 141 & 256 & 402 & 6246 \\
\hline
\end{tabular}
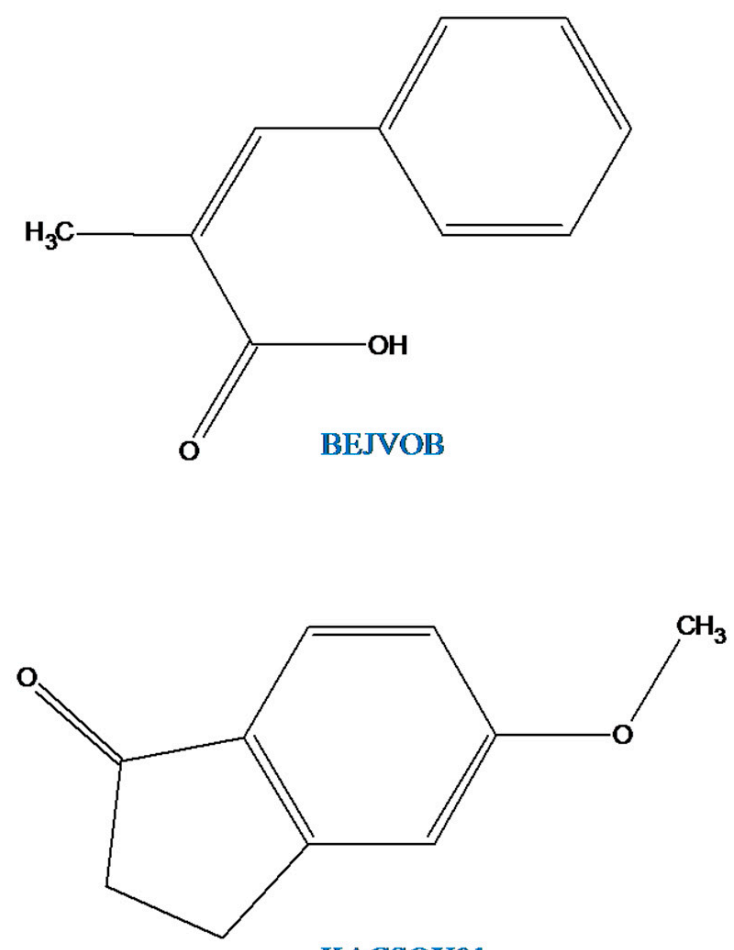

KACSOX01

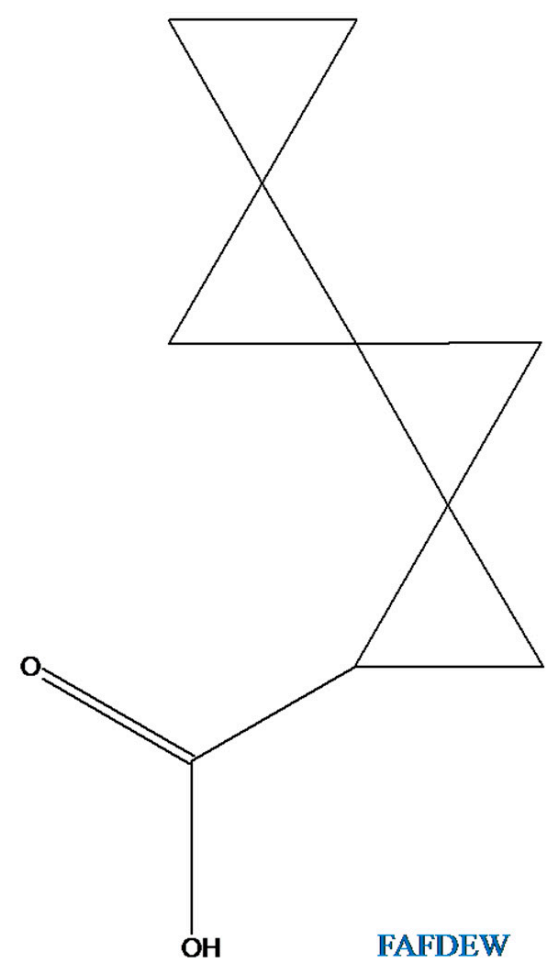

Figure 5. The structural formulas of isomeric $\alpha$-methyl-trans-cinnamic acid (refcode: BEJVOB), 5-methoxyindan-1-one (refcode: KACSOX01), and (1RS,3SR,4SR)-trispiro(2.0.0.2.1.1)nonane-1carboxylic acid (refcode: FAFDEW). 
There are three crystal structures with $\mathrm{CN}_{\mathrm{mol}}=14$, but characterized by the different topological types (Table 3): bcu-x, gpu-x, and tcg-x. Furthermore, all the structures have $\mathrm{CN}_{\text {mol }}{ }^{\prime}=9$ and the same Wyckoff sequence for the midpoints of intermolecular contacts $\left(e^{5} d c b a\right)$. This means the same $H_{\text {edge }}=3.093$ as for the other 2352 structures of the Wyckoff sequence which contains $e^{5}$, including $e^{5} d c b a$ (2324 structures).

Table 3. Topological types and structural characteristics of the molecular net in BEJVOB, KACSOX01, and FAFDEW.

\begin{tabular}{|c|c|c|c|}
\hline Refcode in CSD & BEJVOB [45] & KASSOX01 [46] & FAFDEW [47] \\
\hline Formula & $\mathrm{C}_{10} \mathrm{H}_{10} \mathrm{O}_{2}$ & $\mathrm{C}_{10} \mathrm{H}_{10} \mathrm{O}_{2}$ & $\mathrm{C}_{10} \mathrm{H}_{12} \mathrm{O}_{2}$ \\
\hline Name & $\alpha$-methyl-trans-cinnamic acid & 5-methoxyindan-1-one & $\begin{array}{c}(1 R S, 3 S R, 4 S R)- \\
\text { trispiro(2.0.0.2.1.1)nonane- } \\
\text { 1-carboxylic acid }\end{array}$ \\
\hline Temperature & room & room & $100 \mathrm{~K}$ \\
\hline$R$-factor & 4.10 & 3.90 & 3.75 \\
\hline Structural class & $P 2_{1} / c, Z=4(1)$ & $P 2_{1} / c, Z=4(1)$ & $P 2_{1} / c, Z=4(1)$ \\
\hline \multicolumn{4}{|c|}{ The molecular net } \\
\hline $\mathrm{CN}_{\mathrm{mol}}$ & 14 & 14 & 14 \\
\hline Type (transitivity) & bcu-x $(1,2)$ & gpu-x $(1,4)$ & $\operatorname{tcg}-x(1,6)$ \\
\hline $\mathrm{CN}_{\mathrm{mol}}{ }^{\prime}$ & 9 & 9 & 9 \\
\hline $\begin{array}{c}\text { Wyckoff sequence } \\
\text { of intermolecular contacts }\end{array}$ & $e^{5} d c b a$ & $e^{5} d c b a$ & $e^{5} d c b a$ \\
\hline$H_{\text {edge }}$, bits $/$ contact & 3.093 & 3.093 & 3.093 \\
\hline \multicolumn{4}{|c|}{ The critical net } \\
\hline $\mathrm{CN}_{\text {crit }}$ & 6 & 4 & 5 \\
\hline Type (transitivity) & sxa $(1,3)$ & dia $(1,1)$ & bnn $(1,2)$ \\
\hline $\mathrm{CN}_{\text {crit }}{ }^{\prime}$ & 5 & 3 & 3 \\
\hline $\begin{array}{l}\text { Wyckoff sequence } \\
\text { of bearing contacts }\end{array}$ & $e d c b a$ & $e d c$ & $e^{2} b$ \\
\hline$H_{\text {edge,crit }}$, bits/contact & 2.252 & 1.500 & 1.522 \\
\hline
\end{tabular}

The critical nets for BEJVOB, KACSOX01, and FAFDEW are of different topological type. It was shown in [26] that the value $\Delta=\mathrm{CN}_{\text {crit }}{ }^{\prime}-\operatorname{minCN_{\text {crit}}}{ }^{\prime}$ adopts almost normal discrete distribution, where $92 \%$ of structures demonstrate $\Delta \leq 2$ (for the set of crystalline hydrocarbons this portion was even more $95 \%$ [48]). In the space group $P 2_{1} / c$ there are 3 generators in a minimal generating subset [49]. If a molecule occupies some special position of the space group with a site-symmetry group containing a generating element of the space group ( $\overline{1}$ in $\left.P 2_{1} / c\right)$, then a fewer number of intermolecular contacts along the other symmetry elements could be sufficient for generating of a molecular net. However,

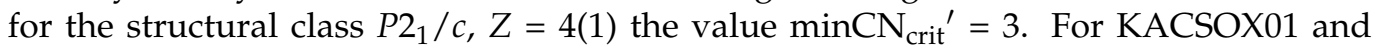
FAFDEW the critical molecular nets are parsimonic $\left(\mathrm{CN}_{\text {crit }}{ }^{\prime}=3\right)$, while for BEJVOB the net is not parsimonic $\left(\mathrm{CN}_{\text {crit }}{ }^{\prime}=5\right)$. The last one contains two redundant contacts via the inversion centers. Any pair of two inversion centers separated by a translation generate this translation; however, if it is accompanied by a contact with the multiplicity 2 along the same direction, the pair of inversion centers becomes redundant. Conversely, a sole contact with the multiplicity 2 in the critical net cannot be redundant because a triplet of inversion centers would never generate a 3D-space group instead of a plane group with the triplet belonging to the plane. As a result of the redundancy the critical net in BEJVOB is more complex than in KACSOX01 and FAFDEW ( $H_{\text {edge,crit }}=2.252,1.500$ and 1.522 bits $/$ atoms, respectively), i.e., about a half of the molecular net information content for KACSOX01 and FAFDEW, and more than 2/3 of that for BEJVOB. The nets are shown in Figure 6. 


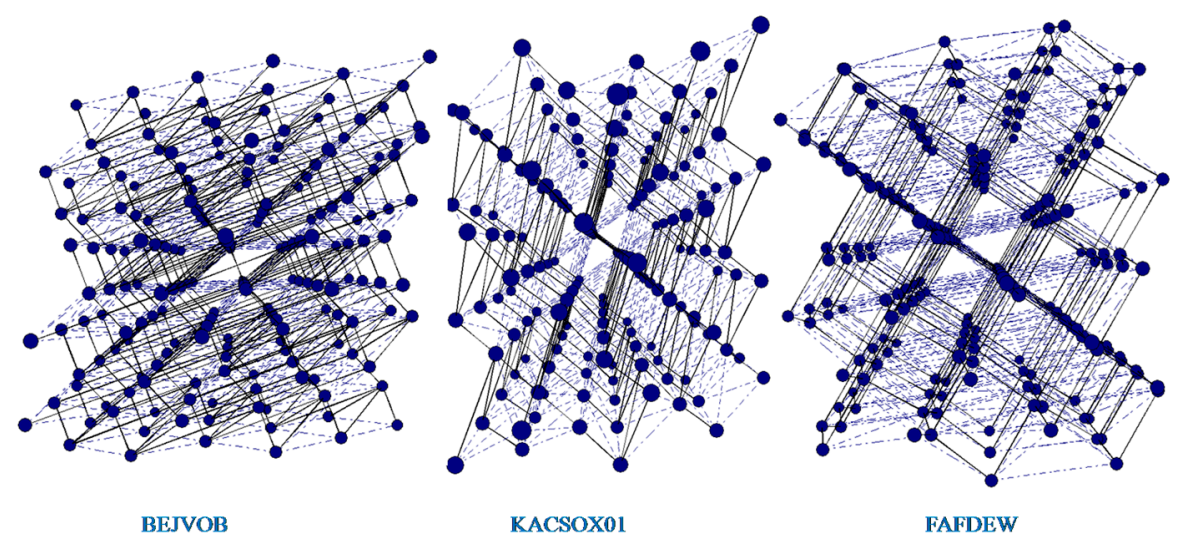

Figure 6. A fragment of the molecular nets of BEJVOB, KACSOX01, and FAFDEW, view along $Y$ ( $\beta$-setting). The edges of the critical nets are shown-by black solid lines, those disposable for the molecular net—by blue dashed lines, the molecular centers of gravity—by blue circles.

The topological types of the molecular and critical nets, which are subnets of the former, are shown in Figure 7. Surprisingly, for BEJVOB the prototype molecular net bcu-x has 2 kinds of edges, while the prototype critical net sxa has three kinds of edges because some Wyckoff positions are split when the symmetry group descends from $\operatorname{Im} \overline{3} m$ (bcu-x) to Cmme (sxa). The group Cmme has five elements in a minimal generating set [49], and there are $Z=4(m m 2)$ equivalent vertices in sxa. As the point group $m m 2$ has two generators, the vertex configuration of sxa can be generated by $5-2=3$ "contacts" of the vertices, therefore, the net sxa could be realized even for $\mathrm{CN}_{\text {crit }}{ }^{\prime}=3$. On the contrary, another similar 6-coordinated net $\mathbf{s x b}$ of the strucutral class $C c c m, Z=4(2 / \mathrm{m})$ could not be realized in any

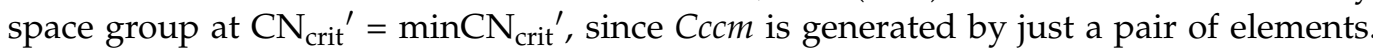
Recently, sxb was found in a metal-organic framework (MOF) $\left[\mathrm{Mg}_{3}(\mathrm{btdc})_{3}(\mathrm{dmf})_{4}\right]$ [50], which was synthesized by a topotactic reaction from $\left[\mathrm{Mg}_{3}(\mathrm{btdc})_{3}(\mathrm{dmf})_{4}\right] \cdot \mathrm{DMF}$ of the pcu type upon heating, thus, the former MOF is not parsimonic in principle.
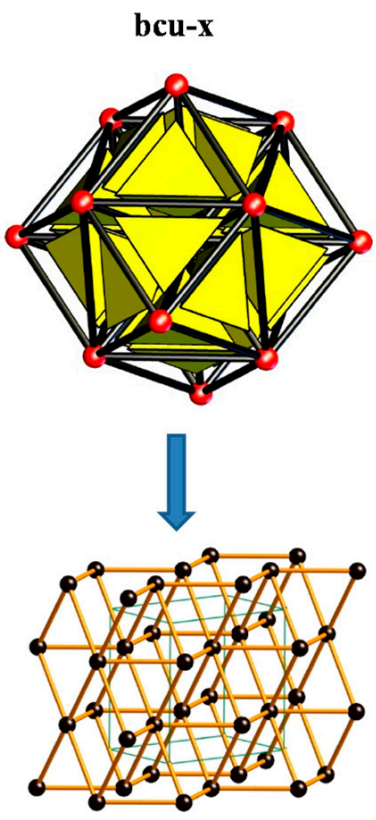

sxa
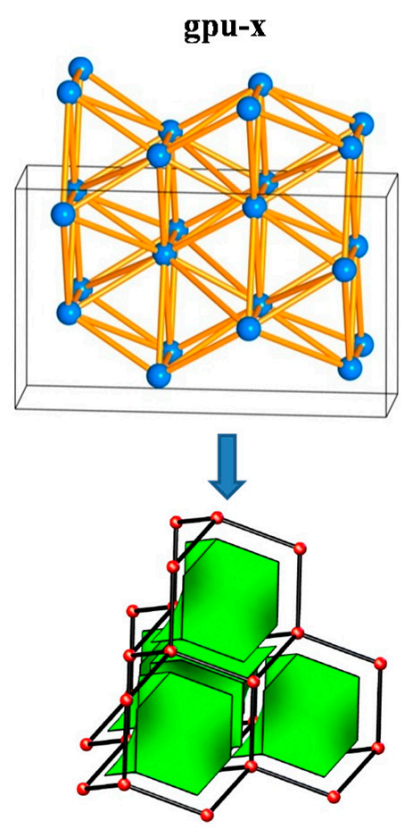

dia

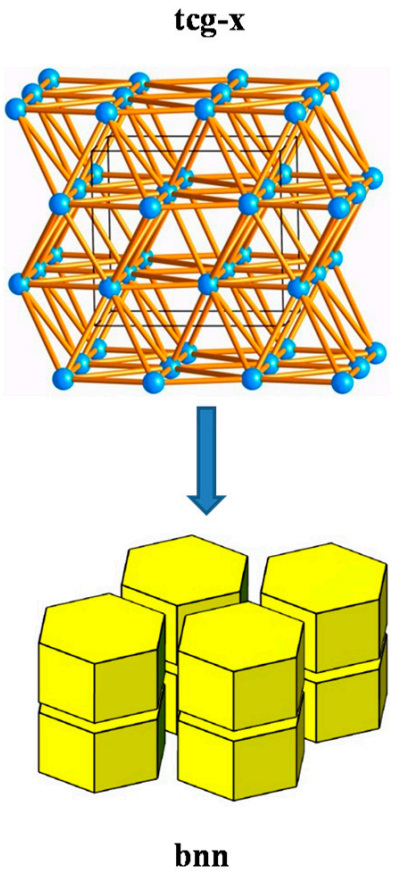

bnn

Figure 7. The topological types of BEJVOB (left), KACSOX01 (center), and FAFDEW (right) for the molecular (top) and critical (bottom) nets. 
The set of the combinatorically distinctive critical nets depends on the topology of the initial molecular nets. For bcu-x, gpu-x, and tcg-x in the three above mentioned crystal

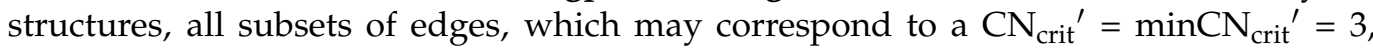
were enumerated (Table 4). As all the initial nets have edges with the Wyckoff sequence $e^{5} d c b a$, there are 4 involutions and 5 contacts with the multiplicity 2 . In BEJVOB (bcu-x), KACSOX01 (gpu-x) and FAFDEW (tcg-x) there are four contacts along the pair of screw axes $2{ }_{1}$, four contacts along the pair of glide $c$-planes, and two contacts along the translation vector, but their combination with the four involutions in different topological types is different. This leads to a different number and types of the critical subnets.

Table 4. Possible critical subnets of $\mathbf{b c u - x}$, gpu-x, and tcg-x with the Wyckoff sequence of edges $e^{5} d c b a$ in $P 2{ }_{1} / c$.

\begin{tabular}{|c|c|c|c|c|c|}
\hline \multirow{2}{*}{$\mathrm{CN}_{\text {crit }}$} & \multirow{2}{*}{$\begin{array}{c}\text { Wyckoff Sequences } \\
\text { of Edges }\end{array}$} & \multirow{2}{*}{ Subnets } & \multicolumn{3}{|c|}{ Nets } \\
\hline & & & Bcu-X & Gpu-X & Tcg-X \\
\hline \multirow{4}{*}{4} & \multirow{4}{*}{$e}$. & dia & 18 & 8 & 20 \\
\hline & & cds & - & 2 & - \\
\hline & & $\mathrm{dmp}$ & - & 4 & - \\
\hline & & 4T19 & - & 2 & - \\
\hline \multirow{4}{*}{5} & \multirow{4}{*}{$e^{2}$. } & sqp & 4 & 4 & 8 \\
\hline & & nov & 8 & 6 & 10 \\
\hline & & bnn & 16 & - & 16 \\
\hline & & $5 \mathrm{~T} 12$ & - & 2 & - \\
\hline \multirow[t]{2}{*}{6} & $e^{3}$. & pcu & 7 & 2 & 8 \\
\hline & Total & & 53 & 30 & 62 \\
\hline
\end{tabular}

Apparently, the complexity of the partition of subnets into the combinatorically distinctive Wyckoff sequences $\left(e, e^{2}\right.$, and $\left.e^{3}\right)$, as well as into the topological types (dia, cds, $\mathbf{d m p}$, etc.), can be easily measured in terms of (1) and (2), but this is out of the topic of this work. In fact, the coordination shell of a molecule may be referred as fuzzy [51], because upon the crystallization different subsets of bearing contacts arise simultaneously. In summary, the subnets of gpu-x are obviously more diverse and include such exotic topological types as 4-coordinated 4T19 (2 subnets) and 5-coordinated 5T12 (2 subnets). Meanwhile, the leading topological type of the subnet in all cases is the diamondoid type dia. In the structural class $P 2_{1} / c, Z=4(1)$ dia, as any other 4-coordinated subnet, is formed by two involutions and two contacts with the multiplicity 2 . The formation of dia is limited by two combinatorically different options [48]. In the first one, the generators are the glide $c$-plane and the inversion centers located at a distance of $b / 4$ from each other along $Y$. The second option entails the screw axis 21 located at the distance of $c / 4$ from one of the inversion centers (Figure 8, top). If one of the inversion centers in the first dia subtype is shifted by $b / 2$, the subnet transforms into the cds type, for the second option it transforms into dmp. The bnn subnet, as dia, exists in two different subtypes (Figure 8, bottom). Each subtype has the only contact via the inversion center and 2 contacts along the translation $a$. The only difference is the last contact with the multiplicity 2, either along the glide $c$-plane or along the screw axis $2_{1}$. However, if the contacts along $a$ are replaced by the contacts along the $2_{1}$ axis located at a distance of $(a / 2+c / 4)$ from the initial inversion center, then the bnn subtypes are transformed into nov and sqp, respectively. Finally, the pcu subnet of each initial net is generated by three contacts with the multiplicity 2 . 

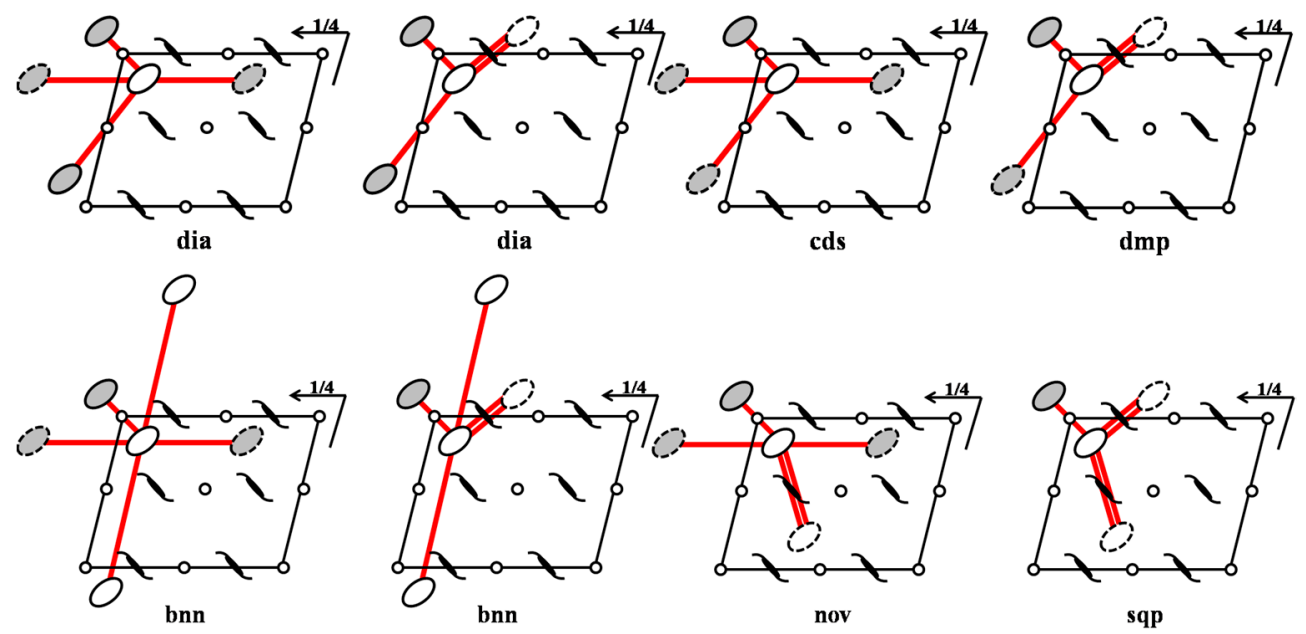

Figure 8. A scheme of the critical subnets of the molecular nets in $P 2{ }_{1} / c, Z=4(1)$ at $\mathrm{CN}_{\text {crit }}=4$ (top) and 5 (bottom). Molecules inverted and turned to the viewer by the reverse side, are shaded up. Molecules shifted by $\pm t / 2$ towards the viewer, are outlined by a dashed line. Double lines denote contacts between the initial molecule and two adjacent ones located above and below.

Of course, among the critical nets in $P 2_{1} / c, Z=4(1)$ there are those having $\mathrm{CN}_{\text {crit }}{ }^{\prime}>\operatorname{minCN} \mathrm{N}_{\text {crit }}{ }^{\prime}$, for instance, noz (5-coordinated), acs, bsn, sxd (6-coordinated), the net of simple hexagonal packing hex (8-coordinated), the body-centered cubic net with unextended coordination shell bcu (8-coordinated), etc. Nevertheless, these topological types may be represented as an extension of some of the 5 minimal nets in $E^{3}$ without collisions and with equal vertex degrees (CNs): dia, cds, ths, pcu, and srs [13]. The last minimal net of this kind, the 3-coordinated srs, was not observed in any crystal structure for the bearing contacts so far. Similarly, the 3-coordinated net ths was not observed in $P 2_{1} / c, Z=4(1)$, but it is possible in some other monoclinic structural classes such as $C 2 / c$, $Z=8(1)$. Up to date, in the TopCryst database it was exemplified not by a molecular crystal, but by a MOF of the crystal structure with the refcode RAGFAJ [52]. The quotient graph of any critical net, including a redundant one, may be derived by an addition of an edge to the undirected quotient graph of some minimal net (Figure 9). Remark that $\Delta=\mathrm{CN}_{\text {crit }}{ }^{\prime}-\min \mathrm{CN}_{\text {crit }}{ }^{\prime}=0$ does not necessarily corresponds to a minimal net, because the deletion of an edge lattice and the deletion of the symmetrically equivalent edges are not exactly the same processes. The deletion of all equivalent edges implies the deletion of translationally equivalent edges, but the converse is not true. As a result, a series of not minimal nets such as bnn, sqp, $\mathbf{d m p}$, nov (Figure 8) also corresponds to $\Delta=0$.

The contribution of $H_{\text {edge,crit }}$ into $H_{\text {edge }}$ varies from 33.9 to $100 \%$ (Table 5). Indeed, there are 4 crystal structures with $\mathrm{CN}_{\text {crit }}=\mathrm{CN}_{\text {mol }}$ and $H_{\text {edge,crit }}=H_{\text {edge, }}$, these are extremal cases with the most redundant critical net. The contribution of $H_{\text {mol }}, H_{\text {edge }}$ and $H\left(2 N, \mathrm{CN}_{\text {mol }}\right)$ into $H_{\text {molNet }}$, is, on the average, is $78.9,9.5$ and $11.6 \%$, respectively, with the value of $\sigma$ being a few percent, i.e., the complexity of the molecular net is substantially defined by the value of $H_{\text {mol }}$, but the impact of $H_{\text {edge }}$ and $H\left(2 N, \mathrm{CN}_{\text {mol }}\right)$ is meaningful. The differences of min and max values for the contributions of $H_{\text {edge }}$ and $H\left(2 N, \mathrm{CN}_{\text {mol }}\right)$ into $H_{\text {molNet }}$ are much more than $\sigma$, that means the outliers being not numerous. The values of SIC, calculated using (7), also show different variances. As it was mentioned above, since there are no atoms in a special position, $\mathrm{SIC}_{\mathrm{mol}}=1$ for all the structures. As the maximal multiplicity of a contact is 2 , theoretically, the minimal $\mathrm{SIC}_{\text {edge, crit }}=-\mathrm{CN}_{\text {crit }} / 2 \cdot 2 / \mathrm{CN}_{\text {crit }} \cdot \log _{2}\left(2 / \mathrm{CN}_{\text {crit }}\right) / \log _{2} \mathrm{CN}_{\text {crit }}=1-1 / \log _{2} \mathrm{CN}_{\text {crit }}$. All the structures with average multiplicity 2 in the set have $\mathrm{CN}_{\text {crit }}=6$, consequently, the minimal $\mathrm{SIC}_{\text {edge,crit }}=1-1 / \log _{2} 6=0.613$. The maximal $\mathrm{SIC}_{\text {edge, crit }}=1$ corresponds to the average multiplicity 1 in the structure of (6-methoxycarbonylmethoxynaphthalen-1-yloxy)acetic acid methyl ester (refcode: KOLRAF) [39] with the Wyckoff sequence of edges $d c b a$ and the critical net dia. The values of $\mathrm{SIC}_{\text {edge }}$ and $\mathrm{SIC}_{\text {molNet }}$ have much smaller $\sigma$ than $\mathrm{SIC}_{\text {edge,crit }}$. 


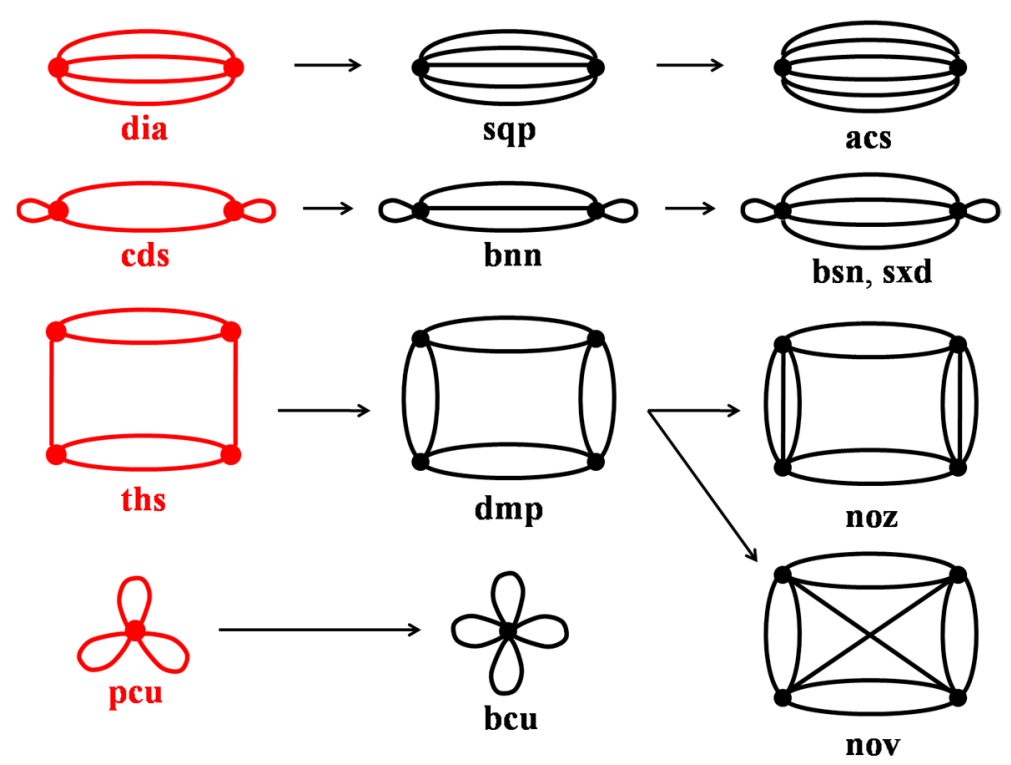

Figure 9. The types of critical subnets in $P 2_{1} / c, Z=4(1)$ derived from minimal nets (shown in red) by an addition of an edge into the quotient graph of the most symmetric embedding in $E^{3}$.

Table 5. The min, max, mean value, and standard deviation of the contributions of $H(\%)$ and SIC for the set of 4152 crystal structures.

\begin{tabular}{|c|c|c|c|c|}
\hline Value & Min & $\operatorname{Max}$ & Mean & $\sigma$ \\
\hline \multicolumn{5}{|c|}{$\%$} \\
\hline$H_{\mathrm{mol}}$ in $H_{\mathrm{molNet}}$ & 35.5 & 94.3 & 78.9 & 6.4 \\
\hline$H_{\text {edge,crit }}$ in $H_{\text {edge }}$ & 41.2 & 100.0 & 62.4 & 11.7 \\
\hline$H_{\text {edge }}$ in $H_{\text {molNet }}$ & 1.9 & 39.2 & 9.5 & 3.6 \\
\hline$H\left(2 N, \mathrm{CN}_{\mathrm{mol}}\right)$ in $H_{\mathrm{molNet}}$ & 3.8 & 25.8 & 11.6 & 2.8 \\
\hline \multicolumn{5}{|c|}{ SIC } \\
\hline $\mathrm{SIC}_{\mathrm{mol}}$ & 1.000 & 1.000 & 1.000 & 0.000 \\
\hline $\mathrm{SIC}_{\text {edge,crit }}$ & 0.613 & 1.000 & 0.740 & 0.057 \\
\hline $\mathrm{SIC}_{\text {edge }}$ & 0.759 & 0.864 & 0.811 & 0.009 \\
\hline $\mathrm{SIC}_{\text {molNet }}$ & 0.813 & 0.879 & 0.853 & 0.008 \\
\hline
\end{tabular}

The distribution of the crystal structures by $H_{\text {mol }}$ and $H_{\text {molNet }}$ is shown in Figure 10. Both values are best approximated by a logistic distribution applicable to the modeling of the degrees of pneumoconiosis in coal miners, chronic obstructive respiratory disease prevalence on smoking, survival time of diagnosed leukemia patients, etc. [53]. Generally, it has the probability density function:

$$
f(x ; \mu ; \beta)=\frac{e^{-(x-\mu) / \beta}}{\beta\left(1+e^{-(x-\mu) / \beta}\right)^{2}}
$$

For $H_{\text {mol }} \mu \approx 5.252, \beta \approx 0.30$; for $H_{\text {molNet }} \mu \approx 5.572, \beta \approx 0.25$. Thus, the difference of the expected values $\mu$ is about 0.320 bits /d.f., and the variance for $H_{\text {mol }}$ is greater than for $H_{\text {molNet }}$.

The discriminatory powers $D$ for $H_{\text {mol }}, H_{\text {edge,crit }}, H_{\text {edge }}, H\left(2 N, \mathrm{CN}_{\text {mol }}\right)$, and $H_{\text {molNet }}$ are listed in Table 6. The simple combinatorial complexity $H_{\mathrm{mol}}$ distinguishes only 99 values, whereas $H_{\text {molNet }}-531$ values. Surprisingly, $H_{\text {edge }}$ has the least $D=0.6372$ and distinguishes only 26 values, while $H_{\text {edge,crit }}$ has even greater $D=0.8762$ and $s=28$. The reason of such substantial difference of $D$ at a small difference of $s$ is the abnormality of distribution. As shown above, 2355 structures have $e^{5} d c b a$ or similar Wyckoff sequence for the edge net (in 
this case $H_{\text {edge }}=1.556$ bits/contact). Meanwhile, the most widespread Wyckoff sequence for the critical net is $e b a$ (or similar) -856 structures $\left(H_{\text {edge,crit }}=1.500\right.$ bits $/$ contact), i.e., with about three times less probability. The $H\left(2 N ; \mathrm{CN}_{\text {mol }}\right)$ has a remarkably high value of $s$ and $D$ in comparison with $H_{\text {mol }}$, because $\mathrm{CN}_{\text {mol }}$ may vary at equal $N$, i.e., at equal $H_{\text {mol }}$.
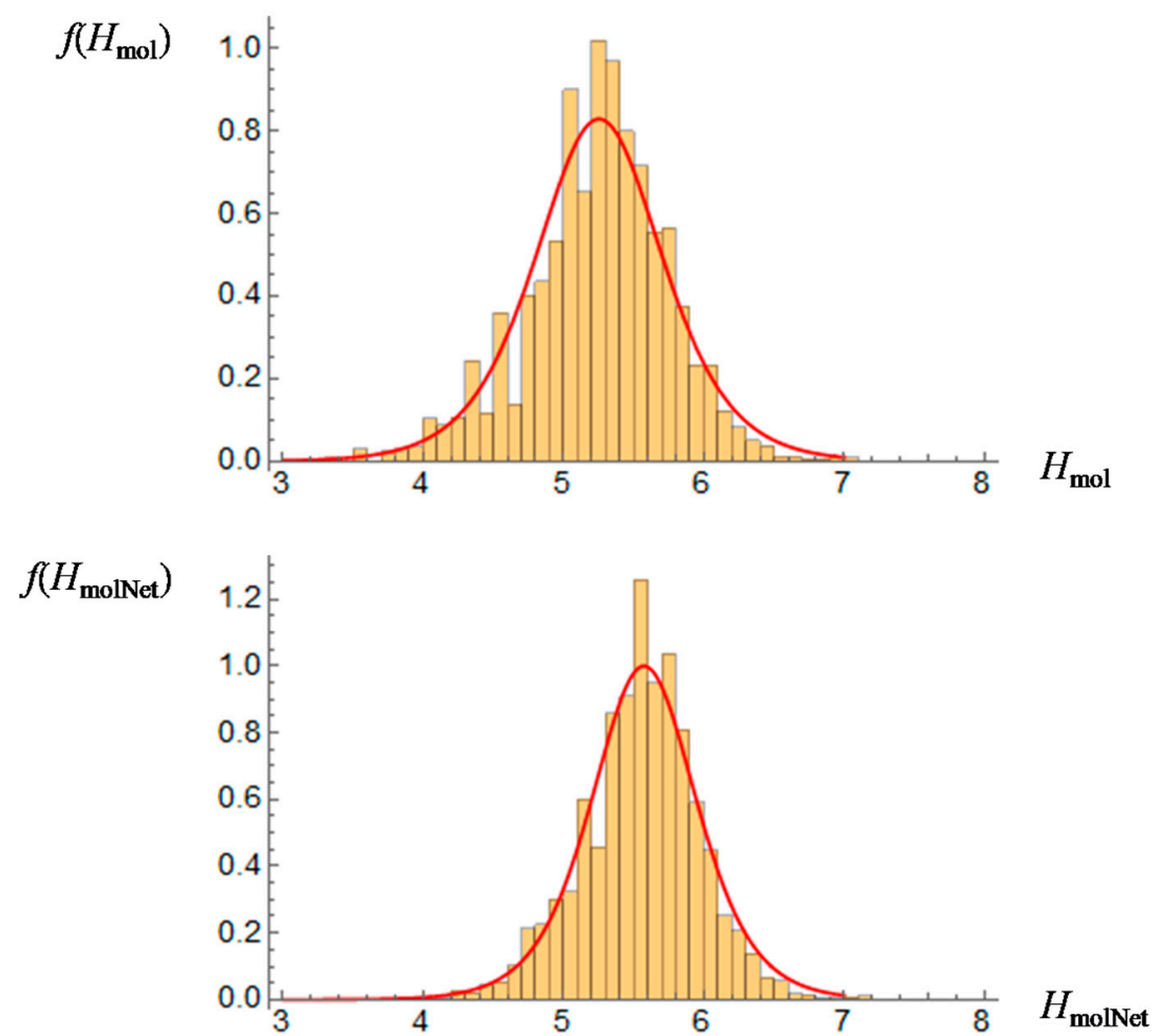

Figure 10. The distributions of 4152 structures by $H_{\text {mol }}$ (top) and $H_{\text {molNet }}$ (bottom) with the step of 0.100 bits/d.f. and their approximation by the logistic distribution.

Table 6. The number $s$ of distinctive values $H$ and the discriminatory power $D$ over the set of 4152 crystal structures.

\begin{tabular}{ccc}
\hline Value & $\boldsymbol{s}$ & $\boldsymbol{D}, \boldsymbol{\%}$ \\
\hline$H_{\text {mol }}$ & 99 & 97.76 \\
$H_{\text {edge,crit }}$ & 28 & 87.62 \\
$H_{\text {egde }}$ & 26 & 63.72 \\
$H\left(2 N ; \mathrm{CN}_{\text {mol }}\right)$ & 389 & 98.93 \\
$H_{\text {molNet }}$ & 531 & 99.13 \\
\hline
\end{tabular}

\section{Conclusions}

For molecular crystals, unlike those with infinite chains, layers, or frameworks, the simple combinatorial information content is of limited usefulness. When each atom occupies its own crystallographic orbit, the value of $H_{\text {mol }}$ reflects only the number of atoms in a molecule. On the contrary, the information content of the molecular net $H_{\text {edge }}$ combined with $H_{\text {mol }}$ gives a hybrid function $H_{\text {molNet }}$ dependent not only on the number of atoms in a molecule, but on the molecular coordination number $\mathrm{CN}_{\mathrm{mol}}$ and the number of orbits occupied by the midpoints of the molecular contacts $\mathrm{CN}_{\mathrm{mol}}{ }^{\prime}$. In comparison with $H_{\text {mol }}$, this hybrid function has a greater discriminatory power and is more favorable for molecular crystals. The edge net complexity $H_{\text {edge }}$ and that originated from mixing two sources of information $H\left(2 N ; \mathrm{CN}_{\text {mol }}\right)$ add, on the average, a little more than $10 \% \mathrm{H}_{\text {mol }}$ each. The normalized values of $H_{\text {edge }}$ and $H_{\text {molNet }}\left(\mathrm{SIC}_{\text {edge }}\right.$ and $\mathrm{SIC}_{\text {molNet }}$ ) are distributed near 
0.80-0.85 with a small standard deviation. The distribution of both $H_{\mathrm{mol}}$ and $H_{\text {molNet }}$ is approximately logistic.

More than a half of 4152 considered structures have $\mathrm{CN}_{\mathrm{mol}}=14$ and $\mathrm{CN}_{\mathrm{mol}}{ }^{\prime}=9$ with the Wyckoff set of edges $e^{5} d c b a$. The average multiplicity of intermolecular contacts statistically significantly decreases upon excluding all contacts except those bearing the molecular net, i.e., the critical net is more saturated with involutions (the inversion centers) than the initial net. The critical net contains more than $40 \%$ information of the molecular net, and $H_{\text {edge,crit }}$ has a more discriminatory power.

The minimal possible $\mathrm{CN}_{\text {crit }}{ }^{\prime}$ is the invariant of a structural class. Each molecular coordination shell may be split in the finite number of critical coordination shells, from which the complexity of the fuzzy coordination shell arises.

Author Contributions: Conceptualization, A.M.B.; methodology, A.M.B.; writing—original draft preparation, A.M.B.; writing-review and editing, A.M.B. and S.M.A. All authors have read and agreed to the published version of the manuscript.

Funding: This research was funded by Russian Science Foundation, grant number 20-77-10065.

Conflicts of Interest: The authors declare no conflict of interest.

\section{References}

1. Shannon, C.E. A Mathematical Theory of Communication. Bell Syst. Tech. J. 1948, 27, 379-423. [CrossRef]

2. Trucco, E. On the information content of graphs: Compound symbols; Different states for each point. Bull. Math. Biophys. 1956, 18, 237-253. [CrossRef]

3. Bonchev, D. Shannon's Information and Complexity. In Complexity: Introduction and Fundamentals; CRC Press: London, UK, 2003; pp. 157-187, ISBN 978-0-429-16545-0.

4. Sabirov, D.S.; Shepelevich, I.S. Information Entropy in Chemistry: An Overview. Entropy 2021, 23, 1240. [CrossRef]

5. Sabirov, D.S. Information entropy changes in chemical reactions. Comput. Theor. Chem. 2018, 1123, 169-179. [CrossRef]

6. Sabirov, D.S. Information entropy of mixing molecules and its application to molecular ensembles and chemical reactions. Comput. Theor. Chem. 2020, 1187, 112933. [CrossRef]

7. Sabirov, D.S. Information entropy of interstellar and circumstellar carbon-containing molecules: Molecular size against structural complexity. Comput. Theor. Chem. 2016, 1097, 83-91. [CrossRef]

8. Chung, S.J.; Hahn, T.; Klee, W.E. Nomenclature and generation of three-periodic nets: The vector method. Acta Crystallogr. Sect. A 1984, 40, 42-50. [CrossRef]

9. Klee, W.E. Crystallographic nets and their quotient graphs. Cryst. Res. Technol. 2004, 39, 959-968. [CrossRef]

10. Eon, J.-G. From symmetry-labeled quotient graphs of crystal nets to coordination sequences. Struct. Chem. 2012, 23, 987-996. [CrossRef]

11. Eon, J.-G. Topological features in crystal structures: A quotient graph assisted analysis of underlying nets and their embeddings. Acta Crystallogr. Sect. A Found. Adv. 2016, 72, 268-293. [CrossRef]

12. Beukemann, A.; Klee, W.E. Minimal nets. Z. Krist.-New Cryst. Struct. 1992, 201, 37-51. [CrossRef]

13. Bonneau, C.; Delgado-Friedrichs, O.; O’Keeffe, M.; Yaghi, O.M. Three-periodic nets and tilings: Minimal nets. Acta Crystallogr. Sect. A Found. Crystallogr. 2004, 60, 517-520. [CrossRef] [PubMed]

14. Eon, J.G. Euclidian embeddings of periodic nets: Definition of a topologically induced complete set of geometric descriptors for crystal structures. Acta Crystallogr. Sect. A Found. Crystallogr. 2011, 67, 68-86. [CrossRef] [PubMed]

15. O'Keeffe, M.; Peskov, M.A.; Ramsden, S.J.; Yaghi, O.M. The Reticular Chemistry Structure Resource (RCSR) Database of, and Symbols for, Crystal Nets. Acc. Chem. Res. 2008, 41, 1782-1789. [CrossRef]

16. Delgado-Friedrichs, O.; O'Keeffe, M. Identification of and symmetry computation for crystal nets. Acta Crystallogr. Sect. A 2003, 59, 351-360. [CrossRef]

17. Sunada, T. Lecture on topological crystallography. Jpn. J. Math. 2012, 39, 1-39. [CrossRef]

18. Krivovichev, S.V. Topological complexity of crystal structures: Quantitative approach. Acta Crystallogr. Sect. A Found. Crystallogr. 2012, 68, 393-398. [CrossRef] [PubMed]

19. Krivovichev, S.V. Which inorganic structures are the most complex? Angew. Chem.-Int. Ed. 2014, 53, 654-661. [CrossRef]

20. Hornfeck, W. On an extension of Krivovichev' s complexity measures. Acta Crystallogr. Sect. A Found. Adv. 2020, 76, 534-548. [CrossRef]

21. Kaußler, C.; Kieslich, G. crystIT: Complexity and configurational entropy of crystal structures via information theory. J. Appl. Crystallogr. 2021, 54, 306-316. [CrossRef] [PubMed]

22. Banaru, A.M.; Aksenov, S.M.; Krivovichev, S.V. Complexity Parameters for Molecular Solids. Symmetry 2021, 13, 1399. [CrossRef]

23. Csiszár, I. Axiomatic Characterizations of Information Measures. Entropy 2008, 10, 261-273. [CrossRef] 
24. Pidcock, E.; Motherwell, W.D.S.; Cole, J.C. A database survey of molecular and crystallographic symmetry. Acta Crystallogr. Sect. B Struct. Sci. 2003, 59, 634-640. [CrossRef] [PubMed]

25. Slovokhotov, Y.L. Organic crystallography: Three decades after Kitaigorodskii. Struct. Chem. 2019, 30, 551-558. [CrossRef]

26. Banaru, A.M.; Aksenov, S.M.; Banaru, D.A. Critical Molecular Coordination Numbers in the Structural Class P2(1)/c, Z = 4(1). Mosc. Univ. Chem. Bull. 2021, 78, 325-333. [CrossRef]

27. Zorky, P.M. Symmetry, pseudosymmetry and hypersymmetry of organic crystals. J. Mol. Struct. 1996, 374, 9-28.

28. Groom, C.R.; Bruno, I.J.; Lightfoot, M.P.; Ward, S.C. The Cambridge Structural Database. Acta Crystallogr. B Struct. Sci. Cryst. Eng. Mater. 2016, 72, 171-179. [CrossRef] [PubMed]

29. Blatov, V.A.; Shevchenko, A.P.; Proserpio, D.M. Applied Topological Analysis of Crystal Structures with the Program Package ToposPro. Cryst. Growth Des. 2014, 14, 3576-3586. [CrossRef]

30. Blatov, V.A. Voronoi-dirichlet polyhedra in crystal chemistry: Theory and applications. Crystallogr. Rev. 2004, 10, 249-318. [CrossRef]

31. Oswald, I.D.H.; Urquhart, A.J. Polymorphism and polymerisation of acrylic and methacrylic acid at high pressure. CrystEngComm 2011, 13, 4503-4507. [CrossRef]

32. Gridin, D.M.; Banaru, A.M. Coordination Numbers and Topology of Crystalline Hydrocarbons. Mosc. Univ. Chem. Bull. 2020, 75, 354-367. [CrossRef]

33. The Samara Topological Data Center TopCryst. Available online: https:/ / topcryst.com/ (accessed on 13 January 2022).

34. Blatov, V.A.; O'Keeffe, M.; Proserpio, D.M. Vertex-, face-, point-, Schläfli-, and Delaney-symbols in nets, polyhedra and tilings: Recommended terminology. CrystEngComm 2010, 12, 44-48. [CrossRef]

35. Hunter, P.R.; Gaston, M.A. Numerical index of the discriminatory ability of typing systems: An application of Simpson's index of diversity. J. Clin. Microbiol. 1988, 26, 2465-2466. [CrossRef]

36. Kroll, L.S. Mathematica-A System for Doing Mathematics by Computer. Wolfram Research. Am. Math. Mon. 1989, 96, 855-861. [CrossRef]

37. Carugo, O.; Blatova, O.A.; Medrish, E.O.; Blatov, V.A.; Proserpio, D.M. Packing topology in crystals of proteins and small molecules: A comparison. Sci. Rep. 2017, 7, 13209. [CrossRef]

38. Starck, F.; Jones, P.G.; Herges, R. Synthesis of Photoresponsive Polyethers. Eur. J. Org. Chem. 1998, 1998, 2533-2539. [CrossRef]

39. Mondal, P.; Karmakar, A.; Singh, W.M.; Baruah, J.B. Crystal packing in some flexible carboxylic acids and esters attached to a naphthalene ring. CrystEngComm 2008, 10, 1550-1559. [CrossRef]

40. Motherwell, W.D.S. Architecture of packing in molecular crystals. CrystEngComm 2017, 19, 6869-6882. [CrossRef]

41. Banaru, A.M.; Gridin, D.M. Coordination Numbers and Critical Topology of Centrosymmetric Hydrocarbons. J. Struct. Chem. 2019, 60, 1885-1895. [CrossRef]

42. Song, X.; Tang, Z.; Zuo, Z.; Duan, J. The crystal structure of 2-(tert-butyl)-4-chloro-6-phenyl-1,3,5-triazine, C13H14Cl1N3. Z. Krist.-New Cryst. Struct. 2018, 233, 779-781. [CrossRef]

43. Boese, R.; Blaeser, D.; Gomann, K.; Brinker, U.H. Spiropentane as a tensile spring. J. Am. Chem. Soc. 1989, 111, 1501-1503. [CrossRef]

44. Shirazi, M.; Soltani, M.-R.; Jahanabadi, Z.; Abdollahifar, M.-A.; Tanideh, N.; Noorafshan, A. Stereological comparison of the effects of pentoxifylline, captopril, simvastatin, and tamoxifen on kidney and bladder structure after partial urethral obstruction in rats. Korean J. Urol. 2014, 55, 756-763. [CrossRef]

45. Bryan, R.F.; White, D.H. $\alpha$-Methyl-trans-cinnamic acid (m.p. 355 K). Acta Crystallogr. Sect. B 1982, 38, 1332-1334. [CrossRef]

46. Abid, O.-R.; Qadeer, G.; Rama, N.H.; Wong, W.-Y. 5-Methoxyindan-1-one. Acta Crystallogr. Sect. E 2007, 63, o165-o166. [CrossRef]

47. de Meijere, A.; Khlebnikov, A.F.; Kozhushkov, S.I.; Kostikov, R.R.; Schreiner, P.R.; Wittkopp, A.; Rinderspacher, C.; Menzel, H.; Yufit, D.S.; Howard, J.A.K. The First Enantiomerically Pure [n]Triangulanes and Analogues: $\sigma$-[n]Helicenes with Remarkable Features. Chem.-Eur. J. 2002, 8, 828-842. [CrossRef]

48. Banaru, A.M.; Banaru, D.A. Zorkii structural classes and critical topology of molecular crystals. J. Struct. Chem. 2020, 61, 1485-1502. [CrossRef]

49. Lord, E.A.; Banaru, A.M. Number of generating elements in space group of a crystal. Mosc. Univ. Chem. Bull. 2012, 67, 50-58. [CrossRef]

50. Dubskikh, V.A.; Lysova, A.A.; Samsonenko, D.G.; Dybtsev, D.N.; Fedin, V.P. Topological polymorphism and temperature-driven topotactic transitions of metal-organic coordination polymers. CrystEngComm 2020, 22, 6295-6301. [CrossRef]

51. Banaru, A.M. A Fuzzy Set of Generating Contacts in a Molecular Agglomerate. Mosc. Univ. Chem. Bull. 2019, 74, 101-105. [CrossRef]

52. Li, S.-L.; Wang, J.; Zhang, F.-Q.; Zhang, X.-M. Light and Heat Dually Responsive Luminescence in Organic Templated CdSO4-type Halogeno(cyano)cuprates with Disorder of Halogenide/Cyanide. Cryst. Growth Des. 2017, 17, 746-752. [CrossRef]

53. Nadarajah, S.; Kotz, S. A generalized logistic distribution. Int. J. Math. Math. Sci. 2005, 2005, 894212. [CrossRef] 